\title{
Effects of the Process Conditions on the formation of the Gas Layer in the Plastic Profile Gas-assisted Co-extrusion Processes
}

\author{
Hesheng Liu, ${ }^{1, a}$, Xiaozhen Deng ${ }^{2, b}$, Xingyuan Huang ${ }^{3, c}$ and Yibin Huang ${ }^{4, d}$ \\ ${ }^{1}$ School of Mechanical and Electronic Engineering, East China Institute of Technology, Jiang Xi, \\ Nanchang 330013,China \\ ${ }^{2}$ Nanchang Institute of Technology, Jiangxi Province Key Laboratory of Precision Drive and \\ Control, Jiang Xi, Nanchang 330099, China \\ ${ }^{3}$ Polymer Processing Research Laboratory of Nanchang University, Jiang Xi, Nanchang 330031 , \\ China \\ ${ }^{4}$ School of Physics and Electronic Information of Shangrao Normal University, Jiang Xi, Shangrao \\ 334001, China \\ aemail: hsliu@vip.163.com, bemail:Pearl617@163.com, cemail: \\ huangxingyuan001@126.com, ${ }^{d}$ email: hyb126@126.com
}

Keywords: Plastic Profile; Gas Layer; Gas-Assisted Co-Extrusion; Experimental Study

Abstract: The gas-assisted co-extrusion molding technology is a new polymer processing molding process integrated with the gas-assisted technology. In the paper, we experimentally explored the effects of several key factors of the gas-assisted co-extrusion molding process with plastic profile on the formation of the stable gas layer. Experimental results showed that in the proper pressure range, the stable gas layer was formed. The relatively lower or higher pressure was not conducive to the formation of the gas layer. The optimal air temperature for the formation of the stable gas layer was close to the melt temperature. In addition, the die temperature, the gas layer thickness, the melt flow, material properties, the external force, and the opening sequence of the extruder screw and pneumatic valve showed the significant effect on the formation of the gas layer.

\section{Introduction}

The conventional co-extrusion molding technology has some disadvantages, such as die swell, large die pressure drop, and melt fracture, which seriously affect the quality of extruded products and its application. Because gas-assisted co-extrusion molding technology can avoid the above disadvantages and improve product quality, it has a good prospect of engineering application. In 1987, the gas-assisted molding technology was first applied in the rubber molding process by Brzoskowski R. et al. [1]. The lubricating effect of gas can effectively reduce die pressure drop. Since then, Huang Li and Qian Bainian $[2,3]$ also successfully applied the gas-assisted molding technology in rubber production. In 2001, Liang RF et al. [4] applied the gas-assisted molding technology in the polymer extrusion process for the first time and found that the technology could effectively relieve the swell phenomenon of extruded products and improve the quality of the extrusion and co-extruded products[5-10].

Current studies of the gas-assisted molding extrusion were mostly focused on numerical simulation and related experimental data were seldom reported. In particular, the effects of the process conditions of the gas-assisted co-extrusion molding on the gas layer were not studied [11-12]. In the paper, we studied the gas-assisted co-extrusion molding processes with L-shaped section profile and experimentally explored the effects of process conditions on the gas layer.

\section{Experimental}

\section{Materials}

Polymer materials used in the experiments include polypropylene (PP) and high-density polyethylene (HDPE) of two different grades. Related properties of used experimental materials are provided in Table 1. 
Table 1 Experimental materials

\begin{tabular}{llllllll}
\hline ID & Polymer & Brand & $\rho\left(\mathrm{g} / \mathrm{cm}^{3}\right)$ & $\begin{array}{l}\text { Melt } \\
\left({ }^{\circ} \mathrm{C}\right)\end{array}$ & $\begin{array}{l}\text { Melt Index } \\
M I(\mathrm{~g} / 10 \mathrm{~min})\end{array}$ & $\eta(\mathrm{Pa} \cdot \mathrm{S})$ & Company \\
\hline 1 & PP & T30S & 0.90 & 164 & 3.2 & 4688 & DaQing \\
2 & PP & $5018 \mathrm{~T}$ & 0.90 & 154 & 1.7 & 8823 & Taiwan \\
3 & HDPE & 8010 & 0.956 & 134 & 1.0 & 15000 & Taiwan \\
\hline
\end{tabular}

\section{Experimental conditions}

In the experiment, the melt temperature and gas temperature were $190{ }^{\circ} \mathrm{C}$. The volumetric flow and pressure of the melt are provided in Table 2.

Table 2 Volumetric flow and pressure of the melt

\begin{tabular}{lll}
\hline Group & $\begin{array}{l}\text { PP(T30S), Volumetric flow and } \\
\text { pressure },\left(\mathrm{cm}^{3} / \mathrm{s}\right) / \mathrm{P}(\mathrm{MPa})\end{array}$ & $\begin{array}{l}\mathrm{PP}(5018 \mathrm{~T}), \text { Volumetric flow and } \\
\text { pressure },\left(\mathrm{cm}^{3} / \mathrm{s}\right) / \mathrm{P}(\mathrm{MPa})\end{array}$ \\
\hline 1st & $0.61 / 0.07$ & $0.78 / 0.10$ \\
2nd & $0.88 / 0.10$ & $1.13 / 0.12$ \\
3rd & $1.13 / 0.14$ & $1.45 / 0.16$ \\
4th & $0.61 / 0.07$ & $1.56 / 0.16$ \\
5th & $0.61 / 0.07$ & $2.34 / 0.22$ \\
\hline
\end{tabular}

\section{Experimental results and analysis}

\section{Effects of the gas pressure}

In the experiment, the gas pressure was gradually increased from 0 to $0.4 \mathrm{MPa}$, and then decreased from 0.4 MPa to 0 . Within a certain pressure range, a stable gas layer between die wall and melt surface was formed and gas-assisted co-extrusion molding was realized. When the gas pressure was relatively low, melt surface was connected with die wall and gas-assisted co-extrusion molding failed. When the gas pressure was relatively high, gas was prone to penetrate the melt to form bubbles and the extruded products might be distorted and deformed, thus leading to the failure of gas-assisted co-extrusion molding. Figure 1(a)-(e) show the effects of the gas pressure on the gas-assisted co-extrusion molding under the prssure of $0 \mathrm{Mpa}, 0.1 \mathrm{Mpa}, 0.2 \mathrm{Mpa}, 0.3 \mathrm{Mpa}$ and $0.4 \mathrm{Mpa}$, respectively.

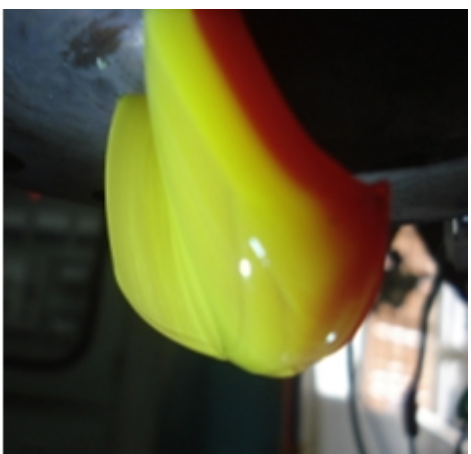

(a) Gas pressure was $0 \mathrm{MPa}$

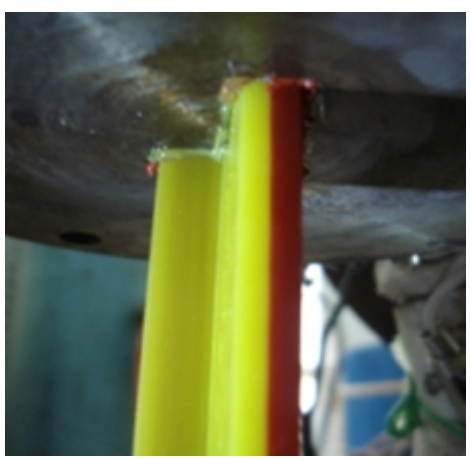

(b) Gas pressure was $0.1 \mathrm{MPa}$

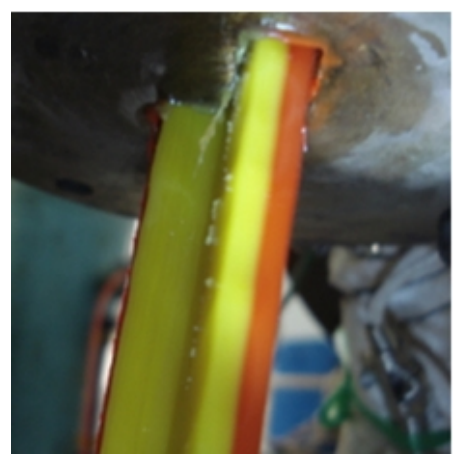

(c) Gas pressure was $0.2 \mathrm{MPa}$ 


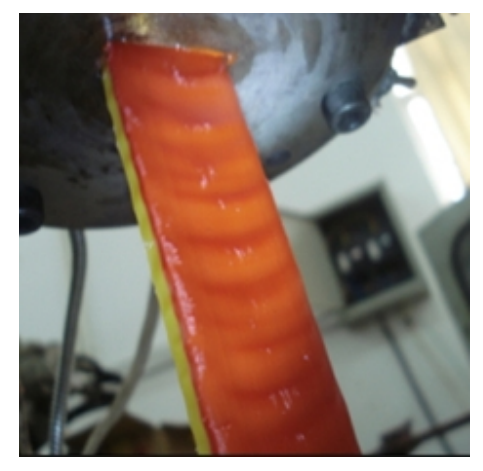

(d) Gas pressure was $0.3 \mathrm{MPa}$

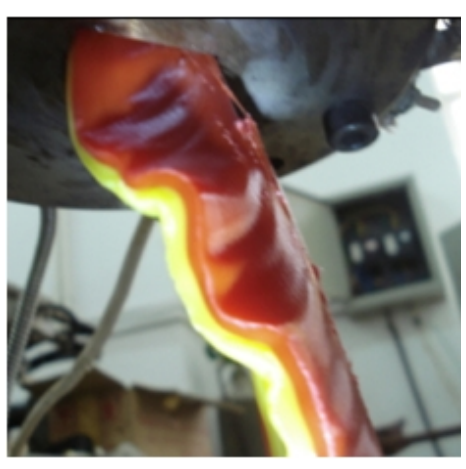

(e) Gas pressure was $0.4 \mathrm{MPa}$

Figure 1 Effects of the gas pressure on the gas-assisted co-extrusion molding

When the gas pressure was 0 , the process was the traditional co-extrusion molding process and obvious swell phenomenon was observed at the die outlet as shown in Figure 1(a). Moreover, the inversion phenomenon from the low-viscosity melt (PP(T30S)) to the high-viscosity melt (PP $(5018 \mathrm{~T})$ ) was also observed. When the gas pressure was increased to $0.1 \mathrm{MPa}$, it was easy to realize the stable gas-assisted co-extrusion molding. The extruded products were soft and the surface was smooth. In figure 1(b), obvious swell phenomenon or the inversion phenomenon was not observed at the die outlet. When the gas pressure was increased to $0.2 \mathrm{MPa}$, the stable gas-assisted co-extrusion molding was realized, as shown in Figure 1(c). When materials were extruded from the die, the slight vibration and roar was heard. The extruded products were hard and slim ripples and white spots could be observed on the surface. The defects might be interpreted as follows. Under the high pressure, gas flow was so strong that the set gas temperature was not reached. The gas at the relatively low temperature might cool the melt surface. Therefore, the extruded products showed the hard texture and white spots. When the gas pressure was increased to $0.3 \mathrm{MPa}$, the stable gas-assisted extrusion molding was realized as shown in Figure 1(d). When the materials were extruded from the die outlet, loud vibration and roar was heard and the extruded products were characterized by the hard texture, large ripples, and significant white spots. When the gas pressure was increased to $0.4 \mathrm{MPa}$, the stable gas-assisted extrusion molding was not realized under the high pressure. The extruded products were distorted and deformed and the formed section was irregular as shown in Figure 1(e).

When the gas pressure was gradually decreased from $0.4 \mathrm{MPa}$ to $0.3 \mathrm{MPa}, 0.2 \mathrm{MPa}$, and $0.1 \mathrm{MPa}$, the stable gas-assisted co-extrusion molding was realized. The extruded products showed the similar surface quality with those obtained during the pressure rise. However, when the gas pressure was decreased the value slightly lower than $0.1 \mathrm{MPa}$, the stable gas-assisted co-extrusion molding was still realized, but the formed gas layer was easily destroyed even by slight external interference. If the gas pressure was continuously decreased, the stable gas-assisted co-extrusion molding was not realized. When the pressure was adjusted to 0 (the pneumatic valve was closed), the process was restored to the traditional co-extrusion molding state.

\section{Effects of the temperature at the die co-extrusion segment}

In the experiment, the temperature at the die co-extrusion segment was respectively set at $180{ }^{\circ} \mathrm{C}$, $190{ }^{\circ} \mathrm{C}$ (the gas temperature), and $200{ }^{\circ} \mathrm{C}$. After the temperature at various die segments reached corresponding set values, extruder screw was started firstly. After melt extrusion was stable, the upper exhaust valve of the gas storage tanker was opened. The heated gas entered die co-extrusion segment through air inlet seam. At the above three temperatures, the stable gas-assisted co-extrusion process was basically the same. When the air pressure was increased to $0.2 \mathrm{MPa}$, the stable gas-assisted co-extrusion was realized within $2 \sim 3 \mathrm{~min}$. The extrusion products obtained at $180{ }^{\circ} \mathrm{C}$ was harder than those obtained at $200{ }^{\circ} \mathrm{C}$. The difference might be interpreted as follows. The lower temperature at the die co-extrusion segment lowered the air temperature. The gas at the lower temperature cooled the melt surface. Therefore, the extrusion products obtained at $180{ }^{\circ} \mathrm{C}$ was harder than those obtained at $200{ }^{\circ} \mathrm{C}$. 


\section{Effects of the gas temperature}

Within the above reasonable air pressure range, the gas temperatures were respectively set at room temperature $\left(36^{\circ} \mathrm{C}\right), 80{ }^{\circ} \mathrm{C}, 120{ }^{\circ} \mathrm{C}, 190{ }^{\circ} \mathrm{C}$ and $200{ }^{\circ} \mathrm{C}$ and then gradually decreased to room temperature. The effects of the gas temperature on the gas-assisted co-extrusion molding are shown in Figure 2.

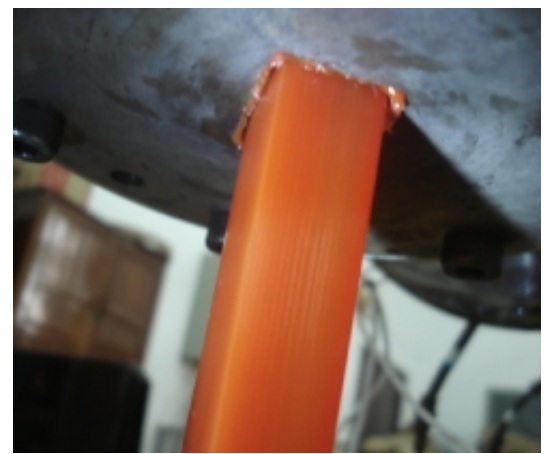

(a) Gas temperature was $200^{\circ} \mathrm{C}$ and $190^{\circ} \mathrm{C}$

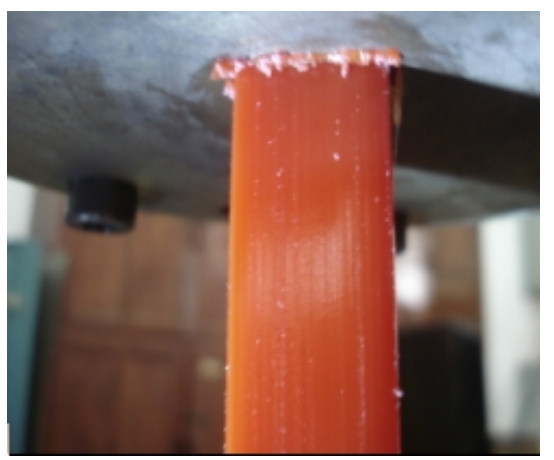

(b) Gas temperature was $120^{\circ} \mathrm{C}$ and $80^{\circ} \mathrm{C}$

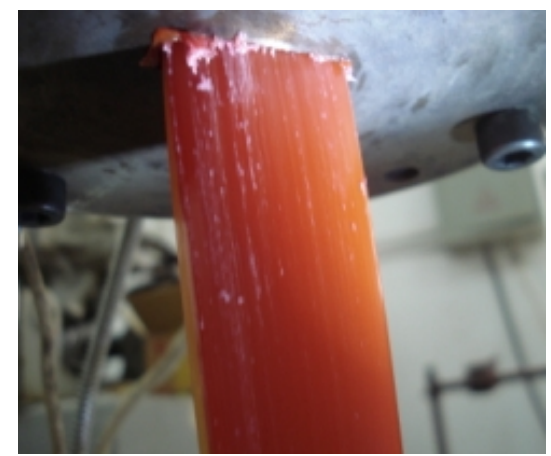

(c) Gas temperature was $36^{\circ} \mathrm{C}$

Figure 2 Effects of the gas temperature on the gas-assisted co-extrusion molding

Under various gas temperatures, the stable gas-assisted co-extrusion could be realized and the optimal gas temperatures were $190{ }^{\circ} \mathrm{C}$ and $200{ }^{\circ} \mathrm{C}$. At $120^{\circ} \mathrm{C}, 80^{\circ} \mathrm{C}$, and $36^{\circ} \mathrm{C}$, it was difficult to realize the stable gas-assisted co-extrusion. The gas pressure should be adjusted to about $0.3 \mathrm{MPa}$ firstly. After residual melts in the air inlet seam were removed by the gas, the gas pressure should be slowly adjusted to $0.1 \mathrm{MPa}$. After the stable gas layer was formed, the extrusion process was stable. The surface quality of the extrusion products was decreased with the decrease in the gas temperature. As shown in Figure 2, when the gas temperature was set to be close to the melt temperature, the extrusion products were characterized by the smooth surface. When the gas temperature was set to be fairly lower than the melt temperature, white spots were observed on the surface of extrusion products. When the gas was not heated, the residual melts around the die outlet edge were bonded on the surface of extrusion products. Therefore, white ripples were observed on the surface of extrusion products. Moreover, when the gas temperature was gradually decreased from $200{ }^{\circ} \mathrm{C}$, the stable gas-assisted co-extrusion was more easily realized. Even though the gas temperature was decreased to room temperature (the heating switch was shut off), the stable gas-assisted co-extrusion was still easily realized. However, extrusion products obtained in the temperature declining stage showed the similar surface quality with those obtained in the temperature rising stage.

\section{Effects of the thickness of the gas layer}

In the experiment of the thickness of gas layer, the gas temperature was set to $190{ }^{\circ} \mathrm{C}$ and the gas pressure was adjusted to about 0.3 $\mathrm{MPa}$. After the extrusion melt was stable, the gas pressure was gradually decreased. The gas layer adopted in the experiment had two different thicknesses: $0.1 \mathrm{~mm}$ and $0.15 \mathrm{~mm}$.

In the experiments with the two thicknesses of the gas layer $(0.1 \mathrm{~mm}$ and $0.15 \mathrm{~mm})$, the minimum gas pressures required for the stable gas-assisted co-extrusion were respectively $0.10 \mathrm{MPa}$ and 0.08 $\mathrm{MPa}$, indicating that the thicker gas layer adopted in the die design required the lower minimum gas pressure.

\section{Effects of melt flow}

As shown in Table 2, the minimum gas pressures required for the stable gas-assisted co-extrusion in the $1^{\text {st }}, 2^{\text {nd }}, 3^{\text {rd }}, 4^{\text {th }}$, and $5^{\text {th }}$ experiments were respectively $0.08 \mathrm{MPa}, 0.10 \mathrm{MPa}, 0.14 \mathrm{MPa}, 0.12 \mathrm{MPa}$, and $0.14 \mathrm{MPa}$. When the two melts had the same melt flow per unit, with the increase in the melt flow, the melt pressure was increased and the minimum gas pressure required for the stable gas-assisted co-extrusion was also increased. The minimum gas pressure required for the stable gas-assisted co-extrusion was basically consistent with the smaller melt pressure in the two melts. When the two melts had different melt flows per unit, with the increase in the melt flow difference, the melt pressure 
difference between the two melts was increased and the minimum gas pressure required for the stable gas-assisted co-extrusion was also increased. The minimum gas pressure required for the stable gas-assisted co-extrusion was between the melt pressure values of the two melts.

\section{Effects of the differences in the properties of experimental materials}

When the two melts had the same melt flow, compared with the melt combination (PP/HDPE), the melt combination (PP/PP) allowed the easier and more stable gas-assisted extrusion process. In the co-extrusion with the melt combination (PP/HDPE), the stable gas-assisted co-extrusion was formed more easily on the PP side than on the HDPE side. After the stable gas-assisted co-extrusion was formed, under the fluctuations of gas flow or melt flow, the stable gas layer on the HDPE side was always destroyed firstly. In the co-extrusion with the melt combination (PP/PP), the formation of the stable gas-assisted co-extrusion showed the wide applicable ranges of temperature and gas pressure. Within a certain range, even the non-heated gas allowed the stable gas-assisted co-extrusion. If residual melts at the die edge could be removed in time, extrusion products with smooth surface could be obtained and the swell phenomenon was not observed. In the co-extrusion with the melt combination (PP/HDPE), the formation of the stable gas-assisted co-extrusion showed the narrow applicable ranges of temperature and gas pressure. When the gas temperature and pressure were respectively close to the temperature and pressure of the melt, the stable gas-assisted co-extrusion could be realized. It was difficult to realize the stable gas-assisted co-extrusion for the melt combination (PP/HDPE).

Moreover, the stable gas layer was easily destroyed and it was difficult to realize long-term stable co-extrusion.

\section{Effects of external forces}

In the experiment of external forces, for the two melt combinations, in the early stage of gas-assisted co-extrusion, the traction force applied on the extrusion products along the co-extrusion direction could promote the formation of the stable gas-assisted co-extrusion, shorten the formation time, and protect the stable gas-assisted co-extrusion from being destroyed by the fluctuations of gas flow and melt flow.

\section{Effects of the opening sequence of screw and pneumatic valve}

To study the effects of the opening sequence of screw and pneumatic valve on the gas-assisted co-extrusion molding, two experiment schemes were adopted. In the first scheme, the extruder screw was started firstly, followed by the pneumatic valve. In the second scheme, the pneumatic valve was opened firstly and the extruder screw was then started. In the first scheme, if the gas pressure was set as $0.1 \mathrm{MPa}$, it was difficult to realize gas-assisted co-extrusion. If the initial gas pressure was set as 0.3 0.4 MPa to purge residual melts in the gas seam and then gradually decreased to $0.1 \mathrm{MPa}$, it was easy to realize the gas-assisted co-extrusion, which was not stable in the initial extrusion stage, as shown in Figure 3(a). Moreover, the roar was heard in the initial stage. Then the gas-assisted co-extrusion became stable and no roar was heard after 2-3 min. In the second scheme, it is easy to realize the gas-assisted co-extrusion. After $1 \mathrm{~min}$, no roar was heard. The initial stage of the gas-assisted co-extrusion was shown in Figure 3(b).

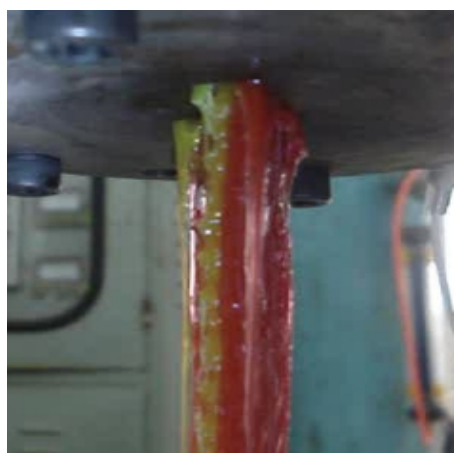

(a) Extruder screw was started firstly

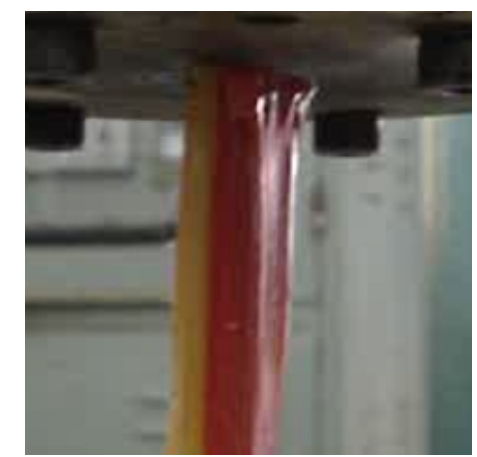

(b) Pneumatic valve was opened firstly

Figure 3 Effects of the opening sequence of screw and pneumatic valve 


\section{Conclusions}

In the gas-assisted co-extrusion with plastic profile, the gas pressure, gas temperature, die temperature, gas layer thickness, melt flow, material properties, external forces, and the opening sequence of screw and the pneumatic valve affect the formation of stable gas layer. In the co-extrusion, if the above factors were controlled within the proper ranges, stable gas-assisted co-extrusion would be realized and superior extrusion products would be obtained. On the contrary, if the above factors were not controlled within the reasonable ranges, the quality of gas-assisted co-extrusion product would be affected and even a stable gas-assisted co-extrusion could not be realized.

\section{Acknowledgements}

This work was financially supported by the National Natural Science Foundation of China (Grant No. 51163011).

\section{References}

[1] R. Brzoskowski, J.L. White, W. Szydlowski, et al. Air-Lubricated Die for Extrusion of Rubber Compounds[J]. Rubber Chemistry \& Technology, 1987, 60(5): 945-956.

[2] R.B. Yoganathan, R. Mammucari, et al. Dense Gas Processing of Polymers[J]. Polymer Reviews,2010, 50(2): 144-177.

[3] B.N. Qian, Y.Q. Tong, C.S. Wang, et al. Air-lubricated Shear Die and Its Application to Short Fibre-reinforced Rubber Hose [J]. China Rubber Industry, 1997, 44(6): 323-326.

[4] R.F Liang, M.R. Mackley. The gas-assisted extrusion of molten polyethylene[J]. Journal Of Rheology, 2001, 45(1): 211-226.

[5] D.R. Arda, M.R. Mackley. Sharkskin instabilities and the effect of slip from gas-assisted extrusion[J]. Rheologica Acta, 2005, 44(4): 352-359.

[6] H.S. Liu, X.Z. Deng, Y.B. Huang, et al. Three-dimensional viscoelastic simulation of the effect of wall slip on encapsulation in the coextrusion process[J]. Journal Of Polymer Engineering, 2013, 33(7): 625-632.

[7] X.Z. Deng, H.S. Liu, Y.B. Huang, et al. Influence of Interface Position on Die Swell in Gas-Assisted Co-Extrusion Molding Processes[J]. Polymer Materials Science And Engineering, 2013, 29(9): 114-118.

[8] Y.B. Huang, H.S. Liu, X.Y. Huang, et al. Finite Element Simulation and Analysis of the Ga-sAssisted Co-Extrusion Interface of Polymer[J]. Polymer Materials Science And Engineering, 2012,28(12): 187-190.

[9] Y.B. Huang, H.S. Liu, X.Y. Huang, et al. Numerical Simulation of the Die Swell in Gas-Assisted Coextrusion [J]. Polymer Materials Science And Engineering, 2010,26(5): 171-174.

[10] X.Y. Huang, H.S. Liu, G.F. Zhou, et al. The Experimental Study of Air-Assisted Extrusion of Polymer [J]. China Plastics, 2005,20(3): 17-19.

[11] X.Y. Huang, H.S. Liu, G. F. Zhou, et al. The influence factors on the set up and stability of gas layer in gas assisted polymer extrusion [J]. Journal Of Plasticity Engineering, 2005,12(5): 101-104 $(+109)$.

[12] Y.B. Huang. Theoretical and Experimental study on polymer gas-assisted co-extrusion [D]. NanChang University, 2011. 